\title{
BMJ Open Global prevalence of diabetic retinopathy: protocol for a systematic review and meta-analysis
}

\author{
Riccardo Cheloni, ${ }^{1,2}$ Stefano A Gandolfi, ${ }^{2}$ Carlo Signorelli, ${ }^{2,3}$ Anna Odone ${ }^{2,3}$
}

To cite: Cheloni R, Gandolfi SA, Signorelli C, et al. Global prevalence of diabetic retinopathy: protocol for a systematic review and meta-analysis. BMJ Open 2019;9:e022188. doi:10.1136/ bmjopen-2018-022188

- Prepublication history and additional material for this paper are available online. To view these files, please visit the journal online (http://dx.doi. org/10.1136/bmjopen-2018022188).

Received 5 February 2018 Revised 12 October 2018 Accepted 3 January 2019

Check for updates

(c) Author(s) (or their employer(s)) 2019. Re-use permitted under CC BY-NC. No commercial re-use. See rights and permissions. Published by BMJ.

${ }^{1}$ IRSO0 - Institute of Research and Study of Optics and Optometry, Vinci, Italy ${ }^{2}$ Department of Medicine and Surgery, University of Parma, Parma, Italy

${ }^{3}$ Faculty of Medicine, University Vita-Salute San Raffaele, Milan, Italy

Correspondence to

Dr Anna Odone;

anna.odone@mail.harvard.edu

\section{ABSTRACT}

Introduction With increasing diabetes trends worldwide, morbidity, mortality and associated costs due to diabetesrelated complications are a global public health concern. Diabetic retinopathy (DR) is among the leading causes of vision loss at the global level; accurate estimates of DR burden is of crucial importance for planning, implementing and evaluating DR prevention and care interventions. The available evidence on DR prevalence at the global level, dating back to 2008 , only considered data from selected regions. Taking into account the rapidly changing patterns in DR epidemiology, the aim of the current study is to carry out a systematic review and meta-analysis to derive solid and updated estimates on global and setting-specific DR prevalence.

Methods and analysis The systematic review methods have been defined following PRISMA guidelines. Studies published from 2008 through 2018 will be identified searching the electronic databases Embase, Medline, Cochrane, ISI Web of Knowledge, as well as through grey literature search. Retrieved records will be independently screened by two authors and relevant data will be extracted from studies reporting data on DR prevalence among individuals with diabetes. Prevalence pooled estimates of any form of DR and vision-threatening DR will be computed applying random-effects meta-analysis. Interstudy heterogeneity will be assessed using the $\mathrm{I}^{2}$ statistic and explored through meta regressions and subgroup analyses. Depending on data availability, we plan to conduct subgroup analyses by study population, diabetes type, DR severity, geographical region and other selected clinical and sociodemographic variables of interest. Quality appraisal of the studies will be performed. Ethics and dissemination Ethics approval is not required as this is a review of anonymised published data. Findings of the final report will be shared with the scientific community through publication in a peer-reviewed journal and presentation at conferences, as well as with key stakeholders, including national and international health authorities, health policy makers, healthcare professionals and the general population.

Clinical trial registration CRD42018085260.

\section{BACKGROUND}

The diabetes epidemic is a major public health concern. ${ }^{1-4}$ Since 1980 age-standardised diabetes mellitus (DM) prevalence has increased by $110 \%$ in men and by $58 \%$

\section{Strengths and limitations of this study}

This systematic review will yield solid and updated estimates on global prevalence of diabetic retinopathy.

- Unlike previous global reports, data included will not be limited to studies conducted in high-income countries and figures from low-income and middle-income countries (LMICs) will be included.

- Increasing data accumulated through screening programmes - in high-income countries as well as in LMICs—will be included in a separate section.

- The dearth of an internationally and widely adopted classification criteria for diabetic macular oedema is likely to reduce the comparability between eligible studies; moreover, it might be a significant cause of heterogeneity.

- In these specific settings, heterogeneity might be caused by a plethora of features; therefore, a good quality of reporting of included studies is crucial in order to proceed with subgroup analysis.

in women, reaching, respectively, $9 \%$ and $7.9 \%$ global prevalence in $2014 .^{1}$ Such an alarmingly high increasing rate, coupled with population growth and ageing, has led to a near quadrupling of the number of adults with diabetes worldwide who are now estimated to be 422 million $^{1}$ and projected to rise to 629 million by $2045 .^{2}$ The highest DM burden is concentrated in low-income and middle-income countries (LMICs).

Diabetic eye disease, resulting from chronic high blood glucose levels causing damage to the retinal capillaries, is the most common microvascular complication of $\mathrm{DM}^{5}$ and includes: diabetic retinopathy (DR) and diabetic macular oedema (DMO). DR is the leading cause of vision loss in working-age adults; ${ }^{6}$ two recent meta-analysis polling data from, respectively, $243^{7}$ and $288^{8}$ population-based studies estimated the global burden of visual impairment and blindness attributable to $\mathrm{DR},{ }^{78}$ reporting that in 2015 2.6 million people were visually impaired because of DR, a figure projected to rise to 
3.2 million in $2020 .^{8}$ Although evidence from epidemiological studies suggest that the incidence of vision-threatening stages of DR is decreasing in high-income countries as a consequence of improved therapies and better DM management, ${ }^{9-11}$ such trends are counterbalanced by increasing DM trends and are not mirrored in LMICs. ${ }^{12} 13$

The latest available estimates of DR prevalence among DM subjects date back to 2008 when DR prevalence data were pooled from 35 studies reporting a global overall prevalence of $34.6 \%$ for any DR, with DR risk increasing with diabetes duration. ${ }^{14}$ There is a strong rationale for updating these data. In fact, in the last decade several factors have contributed to the changing patterns of DR epidemiology, including: DM burden trends, advances in DM and DR diagnosis and treatment, behavioural and population-level DM, and DR risk factors' distribution and healthcare service delivery across different regions. In addition, the above-mentioned meta-analysis by Yau $e t$ $a l^{14}$ did not include data from LMICs where DM burden is highest and access to DR screening and management is lowest. ${ }^{10} 13$

In this context, the aim of the current study is to carry out a systematic review and meta-analysis to derive solid and updated estimates on global and setting-specific (ie, different geographical/regional contexts and subgroups of the population) DR prevalence. These data will inform the planning, implementation and evaluation of DR prevention intervention, and ultimately, contribute to reduce its morbidity in different settings.

\section{METHODS/DESIGN}

As done before ${ }^{15}{ }^{16}$, the review's methods have been defined in advance following the Prepared Items for Systematic Reviews and Meta-Analysis (PRISMA) guidelines $;{ }^{17}$ this protocol was developed according to the PRISMA-P checklist. ${ }^{18}$ (online supplementary appendix $1)$.

The study has been registered on PROSPERO (CRD42018085260).

\section{Criteria for considering studies \\ Study population and study design}

Only studies reporting original data will be included in the review. We will include studies that reported DR or DMO prevalence among representative populations of people with diabetes, or from which prevalence estimates can be derived. We include data on DR prevalence derived from: (1) The general population (ie, from population prevalence surveys).$^{19}$ (2) Populations accessing primary care services (from patients' registers and primary care practices' databases). (3) Hospital-based populations. (4) Screening programmes. We will include studies where DM participants were identified through: positive laboratory tests, physicians' diagnosis, self-reported DM status, DM treatment registries, or other medical or administrative registries. People with both type 1 and type 2 diabetes will be considered. Data from DM in specific populations (eg, patients with severe kidney disease or transplant) will not be excluded but will be considered and analysed in a separate section of the review. No geographical limitations will be applied.

\section{Definitions and outcome measures}

Consistently with Yau et al, ${ }^{14}$ we will consider the following outcomes: (1) Non-proliferative DR (NPDR): every retinopathy graded between levels 20 and 53 (from the Early Treatment Diabetic Retinopathy Study (ETDRS) Severity Scale).$^{20}$ (2) Proliferative DR (PDR): every retinopathy with level $\geq 60$. (3) DMO will be defined as the presence of clinically significant DMO, corresponding to retinal thickening at or within $500 \mu \mathrm{m}$ of the centre of the macula, and/or hard exudates at or within $500 \mu \mathrm{m}$ of the centre of the macula, if associated with thickening of the adjacent retina, and/or a zone or zones of retinal thickening one disc area in size, any part of which is within one disc diameter of the centre of the macula. ${ }^{21}$ Thereafter, composite predefined outcomes will be considered: vision-threatening DR, defined as the presence of PDR and/or DMO, ${ }^{22}$ and any form of DR (any DR), defined as the presence of NPDR, PDR, DMO or any combination thereof. As previously done, outcomes will be considered based on the worse eye or on the single eye affected. ${ }^{14}$ Inclusion will be limited to study providing details on clinical tests used to ascertain DR severity; any grading systems which can be correlated to DR features will be considered and where appropriate, data will be recategorised according to a common definition (see below).

\section{Search methods for identification of studies}

Relevant studies will be identified by searching the electronic databases: Medline, Embase, the Cochrane Library and ISI Web of Science. The search strategy was first developed in Medline using Mesh subject headings combined with free-text terms around the three search components 'Diabetes Mellitus', 'Retinopathy' and 'Prevalence' (online supplementary appendix 2), and then adapted for use in the other databases. Studies published in English from 2008 (the end of the actual study period considered by Yau et $a l^{14}$ ) through March 2018 will be included. In addition, further studies will be retrieved through manual references' listing of included studies and relevant reviews and consultation with experts in the field.

\section{Data collection and analysis \\ Study selection}

Studies retrieved from the different data sources will be grouped together and duplicated records will be removed using a reference management software package (EndNote 2017, Clarative Analytics). Identified studies will be independently reviewed for eligibility by two authors (RC, AO) in a two-step process; a first screen will be performed based on title and abstract while full texts will be retrieved for the second screen. At both stages intercoder agreement will be assessed using Cohen's $\kappa$. 
A minimum $\kappa$ value of 0.75 will be taken to represent high agreement. ${ }^{23}$ Disagreements between reviewers will be resolved by discussion and consultation with a third author (SAG), irrespectively of the $\kappa$ value. The studies' selection process will be graphically reported in a PRISMA flow diagram. ${ }^{17}$

\section{Data extraction and management}

Data will be extracted by one author (RC) supervised by a second author (AO) using a standardised data extraction spreadsheet. The data extraction spreadsheet will be piloted on 10 randomly selected papers and modified accordingly. Authors of the included studies will be contacted in case clarifications or further data are needed (up to three attempts by email). Data extraction will include: authors' names and affiliations, year and country of publication, study design, study setting and country, study period, sample size, sampling method, response/adherence rate, demographic characteristics of study participants (age, gender, ethnicity, etc), type and duration of $\mathrm{DM}, \mathrm{HbAl}_{\mathrm{C}}$ measures, diagnostic methods applied, DR and DMO grading methods or scales, other behavioural and clinical risk factors' distribution (ie, smoking habits, BMI, blood pressure, lipid profile, current use of medications). ${ }^{14}$

DR is a progressive disorder which has been largely classified with respect to its clinical features (see table 1 for a deeper understanding of related clinical signs and classification methods widely used). After a careful extraction of detailed data on diagnosis methods applied in different included studies, data will be recategorised according to common definitions (where applicable), as proposed in table 1 . We plan to apply such a tool to harmonise, as much as possible, severity strata extracted from included studies. We expect a large proportion of included studies to have used the ETDRS grading system. ${ }^{24}$ The 'International Clinical Diabetic Retinopathy and Diabetic Macular Oedema Disease Severity Scale' is a classification more often used in clinical settings, proposed by Wilkinson et al. ${ }^{25}$ Being much simpler and more userfriendly, ${ }^{26}$ we expect its usage to be consistent in studies

Table 1 Summary of features associated with different classification systems of diabetic retinopathy and a proposed equivalence system between three of the most widespread methods worldwide

\begin{tabular}{|c|c|c|c|}
\hline $\begin{array}{l}\text { Disease severity level (from } \\
\text { the international clinical } \\
\text { classification) }^{25}\end{array}$ & Clinical findings & $\begin{array}{l}\text { Derivation from ETDRS } \\
\text { level }^{20}\end{array}$ & $\begin{array}{l}\text { UK screening } \\
\text { programme }^{27}\end{array}$ \\
\hline No apparent retinopathy & No abnormalities & $\begin{array}{l}\text { 10, 14, 15: } \\
\text { DR absent, } \\
\text { DR questionable. }\end{array}$ & Ro \\
\hline Mild NPDR & $\begin{array}{l}\text { Microaneurysms only, or any or all of: } \\
\text { microaneurysms, retinal haemorrhages, } \\
\text { hard exudates, cotton wool spots, up to } \\
\text { the level of moderate NPDR. } \\
\text { No IRMA or significant venous beading. }\end{array}$ & $\begin{array}{l}\text { 20, } 35 \text { A-E: } \\
\text { Very mild NPDR, mild NPDR }\end{array}$ & $\mathrm{R} 1$ \\
\hline Moderate NPDR & $\begin{array}{l}\text { More than just microaneurysms but less } \\
\text { than severe NPDR: } \\
\text { Severe retinal haemorrhages in 1-3 } \\
\text { quadrants or mild IRMA. } \\
\text { Significant venous beading can be } \\
\text { present in no more than one quadrant. } \\
\text { - Cotton wool spots commonly present. }\end{array}$ & $\begin{array}{l}43 \mathrm{~A}-\mathrm{B}, 47 \mathrm{~A}-\mathrm{D} \text { : } \\
\text { moderate NPDR, and } \\
\text { moderately severe NPDR (less } \\
\text { than } 4: 2: 1 \text { ) }\end{array}$ & \\
\hline Severe NPDR & $\begin{array}{l}\text { Any of the following (4-2-1 rule) and no } \\
\text { signs of PDR: } \\
\text { More than } 20 \text { intraretinal haemorrhages } \\
\text { in each of four quadrants. } \\
\text { Definite venous beading in two or more } \\
\text { quadrants. } \\
\text { Prominent IRMA in one or more } \\
\text { quadrants. }\end{array}$ & $\begin{array}{l}53 \text { A-E: } \\
\text { Severe NPDR }\end{array}$ & $\mathrm{R} 2$ \\
\hline PDR & $\begin{array}{l}\text { One or both of the following: } \\
\text { Neovascularisation. } \\
\text { Vitreous and/or preretinal haemorrhage. } \\
\text { Preretinal membrane with/without } \\
\text { tractional retinal detachment. }\end{array}$ & $\begin{array}{l}61,65,71,75,81,85: \\
\text { PDR, high-risk PDR, very } \\
\text { severe or advanced PDR }\end{array}$ & $\begin{array}{l}\text { R3 } \\
\text { (R3A (active) or } \\
\text { R3S (treated and } \\
\text { stable)) }\end{array}$ \\
\hline
\end{tabular}

Adapted from Ting et al (2016) and Scanlon (2017) ${ }^{2628}$

DR, diabetic retinopathy; ETDRS, Early Treatment diabetic Retinopathy Study; IRMA, intraretinal microvascular anomalies; NPDR, nonproliferative DR; PDR, proliferative DR. 
pertaining to clinical environments. A great deal of work in DR grading and standardisation has been performed from the UK's National Health Service diabetic retinopathy screening service. Prior to performing several years of objective fundus evaluation of patients with diabetes in their systematic programmes, a thorough grading system has been approved and widely used in this context. ${ }^{27} 28$ Should included studies have used different scales from those three, we will search for evidence-based corresponding grading with ETDRS. If reclassification from the adopted grading system to the ETDRS, or alternative ones reported in table 1 , will not result possible (eg, inaccurate reporting of diagnosis process, not possible establishing correspondence with widely used and accepted classification system) data will be excluded from the review.

Information on diabetes type will be rigorously extracted. Moreover, as done elsewhere, ${ }^{29}$ we could consider conducting sensitivity analysis applying prevalence rates of types of diabetes of the (matching) general population, when available from the literature and only for included studies where proper sampling was performed (ie, random sampling of the region's households). The differentiation of diabetes type based on the age criterion (ie, type 1 if DM diagnosis occurred before the age of 30 years and type 2 for those diagnosed after the age of 30 years) will be accepted as performed in previous studies. ${ }^{1430}$

\section{Analysis and data synthesis}

We will perform descriptive analysis and report the characteristics of included studies in summary tables and narrative text. As we anticipate variability between included studies, DR prevalence pooled estimates for all prespecified outcomes of interest will be computed applying random-effects meta-analysis models (rather than assuming a single true value in a fixed-effect approach) and reported in forest plots. Meta-analysis will only be limited to studies with at least 200 participants. ${ }^{31}$ Heterogeneity within included studies will be assessed using the $\mathrm{I}^{2}$ statistic and visual inspection of forest plots. ${ }^{32}$ Depending on data availability, we plan to account for heterogeneity conducting-where relevant and possible-meta-regressions and subgroup analysis considering the following covariates: WHO region, World Bank's country income classification, ${ }^{33}$ study period, age group (as per WHO categories), type of diabetes (type 1, type 2), duration of diabetes (in years), $\mathrm{HbAl}_{C}$, other risk factors for which data are available, and DR and DMO diagnosis method. Sensitivity analysis will be carried out considering only studies of the highest methodological quality (ie, meeting seven to nine criteria from the adopted checklist; see below). All analyses will be performed with Stata statistical software V.12 (Stata Corp, College Station, Texas, USA).

\section{Quality assessment}

Quality assessment of included studies will be carried out applying two different instruments: (1) The Joanna Briggs Institute's critical appraisal checklist, specifically developed for studies reporting prevalence data to be included in systematic reviews and meta-analysis (online supplementary appendix 3). ${ }^{34}$ (2) The scorebased quality appraisal tool developed ad hoc by Yau $e t$ $a l^{14}$ and applied in their systematic review and meta-analysis on global DR prevalence (online supplementary appendix 4). The Joanna Briggs Institute's checklist will be used as a reference when conducting sensitivity analysis restricted to high quality studies and, as recently proposed by Tracey et $a \mathrm{l},{ }^{35}$ methodological quality will be considered 'low', 'moderate' and 'high' if three or less, four to six, and seven to nine criteria will be met, respectively.

The quality scale developed by Yau et $a l^{14}$ enters into details of DR diagnosis and DM study populations allowing to qualitatively assess interstudy heterogeneity. However, this instrument presents items that have been self-developed by the same authors. Since this has been identified to be a limit of a great proportion of systematic reviews of the prevalence study, ${ }^{36}$ we plan to use this checklist with the only aim of assessing consistency between the two instruments. Both instruments will be independently applied by two authors (RC, $\mathrm{AO}$ ) and any disagreement will be settled by consensus or adjudication with a third author (SAG), if necessary.

\section{Patient and public involvement}

As this will be a review of published data, patients will not be primarily involved in any stage of the study. Data will be collected from published studies available in the underlined electronic databases.

\section{DISCUSSION}

Taking into account the rapidly changing patterns in DM and DR epidemiology there is a strong rationale for updating current available evidence on global DR prevalence. In fact, as mentioned above, the only systematic review and meta-analysis available in the literature was published in 2012, ${ }^{14}$ reporting data through 2008.

The systematic review and meta-analysis we plan to carry builds on the methodology applied by Yau et $a l^{14}$ so as to produce comparable estimates but, at the same time, adds some substantial improvements, including: the use of a more comprehensive and detailed search strategy and the use of additional sources of data (ie, scientific databases). In addition, differently from Yau $e t a l^{14}$ we will not limit the review to studies conducted in high-income countries but will instead also include data from LMICs which experience the highest DM and DM-associated complications' burden. Last but not the least, we will include, in a separate section of the review, data on DR screening programmes which are accumulating in high-income settings.

To our knowledge this is the first systematic review and meta-analysis protocol aimed at updating Yau et al s $^{14}$ estimates and no other systematic review has been published since then; one review was published in 2015 reporting 
some DR data although not in a systematic way ${ }^{13}$ and one protocol has recently been published describing the plan to carry out a systematic review on DR prevalence, limited to the paediatric and young adult populations. ${ }^{31}$

We strongly believe our data will be of crucial importance for both research and policy. In fact, the data might inform the development of mathematical models or cost-effectiveness analysis to get projections of future DR burden or to anticipate the impact of DR screening programmes or other control interventions; the data might help to identify settings or subgroups of the population where DR is of higher concern and help to set prevention priorities, to optimise resources allocation and orient future research to fill knowledge gaps.

Contributors $\mathrm{AO}$ initially conceived the study. $\mathrm{RC}$ performed the preliminary search. $A 0$ and $R C$ designed the study and produced the first draft of the study which has been consecutively discussed with SAG and CS. The definitive protocol was approved by all authors (AO, RC, SAG, CS).

Funding The authors have not declared a specific grant for this research from any funding agency in the public, commercial or not-for-profit sectors.

Competing interests None declared.

Patient consent for publication Not required.

Provenance and peer review Not commissioned; externally peer reviewed.

Open access This is an open access article distributed in accordance with the Creative Commons Attribution Non Commercial (CC BY-NC 4.0) license, which permits others to distribute, remix, adapt, build upon this work non-commercially, and license their derivative works on different terms, provided the original work is properly cited, appropriate credit is given, any changes made indicated, and the use is non-commercial. See: http://creativecommons.org/licenses/by-nc/4.0/.

\section{REFERENCES}

1. NCD Risk Factor Collaboration (NCD-RisC). Worldwide trends in diabetes since 1980: a pooled analysis of 751 population-based studies with 4.4 million participants. Lancet 2016;387:1513-30.

2. Zhou Y, Zhang B, Hao C, et al. Omentin-A novel adipokine in respiratory diseases. Int J Mol Sci 2017;19.

3. Cavan D, Makaroff L, da Rocha Fernandes J, et al. The diabetic retinopathy barometer study: global perspectives on access to and experiences of diabetic retinopathy screening and treatment. Diabetes Res Clin Pract 2017;129:16-24.

4. Moss SE, Klein R, Klein BE. The 14-year incidence of visual loss in a diabetic population. Ophthalmology 1998;105:998-1003.

5. Antonetti DA, Klein R, Gardner TW. Diabetic retinopathy. N Engl J Med Overseas Ed 2012;366:1227-39.

6. Cheung N, Mitchell P, Wong TY. Diabetic retinopathy. The Lancet 2010;376:124-36.

7. Leasher JL, Bourne RR, Flaxman SR, et al. Global estimates on the number of people blind or visually impaired by diabetic retinopathy: a meta-analysis from 1990 to 2010. Diabetes Care 2016;39:1643-9.

8. Flaxman SR, Bourne RRA, Resnikoff S, et al. Global causes of blindness and distance vision impairment 1990-2020: a systematic review and meta-analysis. Lancet Glob Health 2017;5:e1221-34.

9. Liew G, Wong VW, Ho IV. Mini review: changes in the incidence of and progression to proliferative and sight-threatening diabetic retinopathy over the last 30 years. Ophthalmic Epidemiol 2017;24:73-80.

10. Sabanayagam C, Yip W, Ting DS, et al. Ten emerging trends in the epidemiology of diabetic retinopathy. Ophthalmic Epidemiol 2016;23:209-22.

11. Wong TY, Mwamburi M, Klein R, et al. Rates of progression in diabetic retinopathy during different time periods: a systematic review and meta-analysis. Diabetes Care 2009;32:2307-13.
12. Klein $\mathrm{R}$, Klein $\mathrm{BE}$. Is the prevalence of visual impairment rising or falling in the people with diabetes mellitus? It depends on who you study. JAMA Ophthalmol 2013;131:948-50.

13. Lee R, Wong TY, Sabanayagam C. Epidemiology of diabetic retinopathy, diabetic macular edema and related vision loss. Eye Vis 2015;2:17.

14. Yau JW, Rogers SL, Kawasaki R, et al. Global prevalence and major risk factors of diabetic retinopathy. Diabetes Care 2012;35:556-64.

15. Amerio A, Tonna M, Odone A, et al. Heredity in comorbid bipolar disorder and obsessive-compulsive disorder patients. Shanghai Arch Psychiatry 2015;27:307-10.

16. Tonna M, Amerio A, Odone A, et al. Comorbid bipolar disorder and obsessive-compulsive disorder: Which came first? Aust N Z J Psychiatry 2016;50:695-8.

17. Moher D, Liberati A, Tetzlaff J, et al. Preferred reporting items for systematic reviews and meta-analyses: the PRISMA statement. Int $J$ Surg 2010;8:336-41.

18. Moher D, Shamseer $L$, Clarke $M$, et al. Preferred reporting items for systematic review and meta-analysis protocols (PRISMA-P) 2015 statement. Syst Rev 2015;4:1.

19. Ruta LM, Magliano DJ, Lemesurier R, et al. Prevalence of diabetic retinopathy in Type 2 diabetes in developing and developed countries. Diabet Med 2013;30:387-98.

20. Early Treatment Diabetic Retinopathy Study Research Group. Fundus photographic risk factors for progression of diabetic retinopathy. Ophthalmology 1991;98:823-33.

21. Kinyoun J, Barton F, Fisher M, et al. Detection of diabetic macular edema. Ophthalmoscopy versus photography--Early Treatment Diabetic Retinopathy Study Report Number 5. The ETDRS research group. Ophthalmology 1989;96:746-50.

22. Zhang X, Saaddine JB, Chou CF, et al. Prevalence of diabetic retinopathy in the United States, 2005-2008. JAMA 2010;304:649-56.

23. Landis JR, Koch GG. The measurement of observer agreement for categorical data. Biometrics 1977;33:159-74.

24. Davis MD, Hubbard LD, Trautman J, et al. Conference on insulin pump therapy in diabetes. Multicenter study effect on microvascular disease. Studies of retinopathy. Methodology for assessment and classification with fundus photographs. Diabetes 1985;34:42-9.

25. Wilkinson CP, Ferris FL, Klein RE, et al. Proposed international clinical diabetic retinopathy and diabetic macular edema disease severity scales. Ophthalmology 2003;110:1677-82.

26. Ting DS, Cheung GC, Wong TY. Diabetic retinopathy: global prevalence, major risk factors, screening practices and public health challenges: a review. Clin Exp Ophthalmol 2016;44:260-77.

27. Harding S, Greenwood R, Aldington S, et al. Grading and disease management in national screening for diabetic retinopathy in England and Wales. Diabet Med 2003;20:965-71.

28. Scanlon $\mathrm{PH}$. The english national screening programme for diabetic retinopathy 2003-2016. Acta Diabetol 2017:54:515-25.

29. Song P, Yu J, Chan KY, et al. Prevalence, risk factors and burden of diabetic retinopathy in China: a systematic review and meta-analysis. $J$ Glob Health 2018;8:010803.

30. Klein R, Klein BE, Moss SE, et al. The Wisconsin epidemiologic study of diabetic retinopathy. III. Prevalence and risk of diabetic retinopathy when age at diagnosis is 30 or more years. Arch Ophthalmol 1984;102:527-32.

31. Ibanez-Bruron MC, Solebo AL, Cumberland PM, et al. Screening for diabetic retinopathy in children and young people in the UK: potentia gaps in ascertainment of those at risk. Diabet Med 2017;34:1012-3.

32. Higgins JP, Thompson SG. Quantifying heterogeneity in a metaanalysis. Stat Med 2002;21:1539-58

33. Zhang R, Zhuang L, Li M, et al. Arg913Gln of SLC12A3 gene promotes development and progression of end-stage renal disease in Chinese type 2 diabetes mellitus. Mol Cell Biochem 2018;437:203-10.

34. Munn Z, Moola S, Lisy K, et al. Methodological guidance for systematic reviews of observational epidemiological studies reporting prevalence and cumulative incidence data. Int J Evid Based Healthc 2015;13:147-53.

35. Tracey ML, Gilmartin M, O'Neill K, et al. Epidemiology of diabetes and complications among adults in the republic of ireland 19982015: a systematic review and meta-analysis. BMC Public Health 2016;16:132.

36. Munn Z, Moola S, Riitano D, et al. The development of a critical appraisal tool for use in systematic reviews addressing questions of prevalence. Int J Health Policy Manag 2014;3:123-8. 